\title{
Neonatal RDS and LUS, is the debate still open?
}

\author{
Alessandro Perri ${ }^{1}$, Milena Tana ${ }^{1}$, and Giovanni Vento ${ }^{2}$ \\ ${ }^{1}$ Department of Woman and Child Health and Public Health, Child Health Area; \\ Fondazione Policlinico Universitario A. Gemelli, IRCCS \\ ${ }^{2}$ ondazione Policlinico Universitario A. Gemelli IRCCS, Roma, Italia.
}

July 7,2020

\begin{abstract}
We read the letter to the editor "B-lines score: artifacts as a sign of neonatal specific disease?" by Quarato et al. and we are pleased by the interest aroused by our article "Neonatal lung ultrasonography score after surfactant in preterm infants: A prospective observational study" published on your journal. This study included preterm neonates with respiratory distress syndrome (RDS), requiring non-invasive ventilation and surfactant. The aim of our citated study was to asses changes of a validated neonatal lung ultrasonography score (nLUS) after surfactant treatment. Our data demonstrate a lowering of the nLUS $2 \mathrm{~h}$ and $12 \mathrm{~h}$ after surfactant treatment. In their letter to the editor Quarato et al. expressed criticism about the nLUS score validation and about the utility of the Lung Ultrasound (LUS) as a diagnostic tool. They conclude that "LUS can be used only for diagnosing minimal pleural effusion and, at least, as complementary imaging, in addiction to chest radiographs $(\mathrm{CR})$, for monitoring the reduction of subpleural pneumonitic consolidations under therapy". Our citated study hasn't focused on validation of the nLUS score or on LUS as a diagnostic tool for neonatal RDS, so we don't get how Quarato's concerns can be addressed to our paper. Nevertheless, finding the debate about nLUS or LUS in the neonatal field an occasion to promote an improving in the care of the preterm babies, we will discuss objections raised in Quadrato's work, point by point.
\end{abstract}

Neonatal RDS and LUS, is the debate still open?

Dear Editor,

we read the letter to the editor "B-lines score: artifacts as a sign of neonatal specific disease?" by Quarato et al. and we are pleased by the interest aroused by our article "Neonatal lung ultrasonography score after surfactant in preterm infants: A prospective observational study" published on your journal ${ }^{1}$. This study included preterm neonates with respiratory distress syndrome (RDS), requiring non-invasive ventilation and surfactant. The aim of our citated study was to asses changes of a validated neonatal lung ultrasonography score (nLUS) after surfactant treatment. Our data demonstrate a lowering of the nLUS $2 \mathrm{~h}$ and $12 \mathrm{~h}$ after surfactant treatment.

In their letter to the editor Quarato et al. expressed criticism about the nLUS score validation and about the utility of the Lung Ultrasound (LUS) as a diagnostic tool. They conclude that "LUS can be used only for diagnosing minimal pleural effusion and, at least, as complementary imaging, in addiction to chest radiographs (CR), for monitoring the reduction of subpleural pneumonitic consolidations under therapy".

Our citated study hasn't focused on validation of the nLUS score or on LUS as a diagnostic tool for neonatal RDS, so we don't get how Quarato's concerns can be addressed to our paper. Nevertheless, finding the debate about nLUS or LUS in the neonatal field an occasion to promote an improving in the care of the preterm babies, we will discuss objections raised in Quadrato's work, point by point.

First of all: Quarato et al. claim that B-lines can be present in a normal lung. Coalescent B-lines wouldn't distinguish neonatal RDS from fibrosis, severe fluid overload due to congenital heart disease or renal failure. 
Moreover, B-lines are present in hydropneumothorax, subpleural cysts, blebs, emphysema, minimal pleural effusion and residual cavity of pneumectomy. This leading to the conclusion that we should not use LUS in management of neonatal RDS.

In our neonatal intensive care unit (NICU) RDS is managed following European Consensus Guidelines on the Management of Respiratory Distress Syndrome - 2019 Update $^{2}$ and we agree with the statement "LUS may be a useful adjunct to clinical decision making, with RDS lungs having a specific appearance that can be differentiated from other common neonatal respiratory disorders and it has potential to reduce Xray exposure" 2 . We assert that after a clinical suspicion of a specific disease, laboratory and instrumental tests, including LUS, are tools that can be used by the neonatologist both to confirm the diagnosis and to exclude other pathological conditions. Surfactant, in a neonate of few hours of life, is administrated considering gestational age and $\mathrm{FiO}_{2}$ and guidelines, in selected situation, don't exclude a clinical diagnosis, not requiring instrumental tests, to guide the therapy ${ }^{2}$. Clearly, diagnosis as fluid overload due to renal or heart disease, fibrosis, residual cavity of pneumectomy are not a usual differential diagnosis of the neonatal RDS and should be considered because of pre-postnatal clinical history or specific physical/laboratory signs (eg. congenital heart disease suspicion won't be formulated by CR or LUS but by cyanosis unresponsive to oxygen therapy or blood gas analysis showing low $\mathrm{PaO}_{2}$ with a normal or low $\mathrm{PaCO} 2$ ).

Another point by Quarato et al. was the intra and inter-operator variability of the nLUS. They also stated that some artifacts used to produce the score could be due to the type of probe used or to the inappropriate set-up of the sonographer. Furthermore, the nLUS is criticised as it would investigate not more than $70 \%$ of pleural surface.

It is known the nLUS has been validated in a previous study ${ }^{3}$ and neonatal LUS agreement between operators has been tested too ${ }^{4,5}$. Previous validation of the score was the reason for us to use it in our study. Both type of probe and ultrasound machine settings are defined in the "method" section of our study. We don't deny some limits of the nLUS, like not resulting from a complete study of the pleural surface. The decision not to extend LUS study to the posterior hemithorax has been motivated with the NICU setting, being neonates requiring intensive care instable. Moreover, concerns about the setting of the sonographer are not realistic, as it should be clear that LUS has to be performed only by trained operators (eg. an image like fig.1b presented by Quarato et al should be immediately recognised as inadequate by the physician and cannot be stored in the clinical records or used to take a clinical decision).

Furthermore, it is a Quarato et al. opinion that LUS is not able to manage a specific disease like RDS. The authors motivated this position reporting a review by Hiles et $\mathrm{al}^{6}$. They also nominated some guidelines in order to present no international acknowledgement for recommending LUS for the diagnosis of neonatal RDS. The review presented by Quarato et al, focused on chest x-ray vs LUS, is quite outdated, being published on 2016 and missing some studies like these ${ }^{7-9}$. A more recent review and meta-analysis ${ }^{10}$ state that "LUS, particularly LUS score, can be used accurately to determine the need for surfactant replacement treatment or mechanical ventilation in infants with respiratory distress treated with nCPAP support". About the nominated guidelines, authors decided not to cite them. So, it could be our mistake, but we couldn't find (not even in one of them) the paragraphs focusing on the neonatal RDS. In our NICU, anyway, LUS is performed following these guidelines ${ }^{11}$ and protocol ${ }^{12}$, which are focused on the neonate's disease. Differential patterns of LUS referring to the RDS are presented, being specific for the detection of: transient tachypnea of the newborn, RDS, meconium aspiration syndrome, pneumonia, atelectasis, pneumothorax, pleural effusion and normal lung.

A sentence about surfactant replacement treatment not being cause of changes on the neonatal lung visible on LUS is reported by Quarato et al. The sentence was due to an interesting study of Cattarossi et al ${ }^{13}$, published in 2010. Key message is that surfactant resolves regional alveolar aeration, but it does not affect total lung fluid content. We shared this message in our study. For this reason, we analysed not only nLUS, but also LUS patterns changes $2 \mathrm{~h}$ and $12 \mathrm{~h}$ after surfactant administration. We showed how LUS patterns don't change immediately after surfactant therapy. As stated in "conclusion" section of our study, this data could be due to a wide nLUS variation (0-18) that permits to detect even little changes in lung fluids, 
undetectable performing a global analysis of LUS patterns. Another possibility is that nLUS changes with an increase in aeration of the lung, being this situation demonstrated, in adult, in positive end expiratory pressure induced lung recruitment ${ }^{14}$.

We thank Quarato et al. to have focused on nLUS and LUS, giving us the opportunity to discuss about them. We want to remark a data taken from a study demonstrating the reduction of radiation exposure in "A.Béclère Medical Center", Paris, France, with the use of LUS in NICU from $2014^{15}$. The average number of chest X-rays per baby and the mean radiation dose per baby were calculated before the introduction of LUS. Chest X-ray entrance surface dose was 28, 30 and $32 \mu \mathrm{Gy}$ for babies weighing less than $1500 \mathrm{~g}$, 1500-2500 g and more than $2500 \mathrm{~g}$, respectively. The Reduction of radiation exposure should be a goal in a NICU setting, because of the vulnerability of premature patients even to radiation. Both the nLUS and LUS represent interesting subjects in the neonatal research field. Nevertheless, we consider LUS a tool in the ordinary evaluation of a neonate clinically diagnosed with RDS, according to the most recent European guidelines ${ }^{2}$.

1. Perri A, Tana M, Riccardi R, et al. Neonatal lung ultrasonography score after surfactant in preterm infants: A prospective observational study. Pediatr Pulmonol . 2020;55(1):116-121. doi:10.1002/ppul.24566

2. Sweet DG, Carnielli V, Greisen G, et al. European Consensus Guidelines on the Management of Respiratory Distress Syndrome - 2019 Update. Neonatology . 2019;115(4):432-450. doi:10.1159/000499361

3. Brat R, Yousef N, Klifa R, Reynaud S, Shankar Aguilera S, De Luca D. Lung Ultrasonography Score to Evaluate Oxygenation and Surfactant Need in Neonates Treated With Continuous Positive Airway Pressure. JAMA Pediatr . 2015;169(8):e151797. doi:10.1001/jamapediatrics.2015.1797

4. Brusa G, Savoia M, Vergine M, Bon A, Copetti R, Cattarossi L. Neonatal Lung Sonography: Interobserver Agreement Between Physician Interpreters With Varying Levels of Experience. J Ultrasound Med . 2015;34(9):1549-1554. doi:10.7863/ultra.15.14.08016

5. Raimondi F, Migliaro F, Sodano A, et al. Use of neonatal chest ultrasound to predict noninvasive ventilation failure. Pediatrics . 2014;134(4):e1089-e1094. doi:10.1542/peds.2013-3924

6. Hiles M, Culpan AM, Watts C, Munyombwe T, Wolstenhulme S. Neonatal respiratory distress syndrome: Chest X-ray or lung ultrasound? A systematic review. Ultrasound . 2017;25(2):80-91. doi:10.1177/1742271X16689374

7. Corsini I, Parri N, Gozzini E, et al. Lung Ultrasound for the Differential Diagnosis of Respiratory Distress in Neonates. Neonatology . 2019;115(1):77-84. doi:10.1159/000493001

8. De Martino L, Yousef N, Ben-Ammar R, Raimondi F, Shankar-Aguilera S, De Luca D. Lung Ultrasound Score Predicts Surfactant Need in Extremely Preterm Neonates. Pediatrics . 2018;142(3):e20180463. doi:10.1542/peds.2018-0463

9. Perri A, Riccardi R, Iannotta R, et al. Lung ultrasonography score versus chest X-ray score to predict surfactant administration in newborns with respiratory distress syndrome. Pediatr Pulmonol . 2018;53(9):1231-1236. doi:10.1002/ppul.24076

10. Razak A, Faden M. Neonatal lung ultrasonography to evaluate need for surfactant or mechanical ventilation: a systematic review and meta-analysis. Arch Dis Child Fetal Neonatal Ed . 2020;105(2):164-171. doi:10.1136/archdischild-2019-316832

11. Kurepa D, Zaghloul N, Watkins L, Liu J. Neonatal lung ultrasound exam guidelines. J Perinatol . 2018;38(1):11-22. doi:10.1038/jp.2017.140

12. Liu J, Copetti R, Sorantin E, et al. Protocol and Guidelines for Point-of-Care Lung Ultrasound in Diagnosing Neonatal Pulmonary Diseases Based on International Expert Consensus. J Vis Exp . 2019;(145):10.3791/58990. Published 2019 Mar 6. doi:10.3791/58990

13. Cattarossi L, Copetti R, Poskurica B, Miserocchi G. Surfactant administration for neonatal respiratory distress does not improve lung interstitial fluid clearance: echographic and experimental evidence. $J$ Perinat Med . 2010;38(5):557-563. doi:10.1515/jpm.2010.096

14. Bouhemad B, Brisson H, Le-Guen M, Arbelot C, Lu Q, Rouby JJ. Bedside ultrasound assessment of positive end-expiratory pressure-induced lung recruitment. Am J Respir Crit Care Med . 
2011;183(3):341-347. doi:10.1164/rccm.201003-0369OC

15. Shen P, Luo R, Gao Y, Wang J, Zhang M. Zhonghua Jie He He Hu Xi Za Zhi . 2014;37(5):332336. Escourrou G, De Luca D. Lung ultrasound decreased radiation exposure in preterm infants in a neonatal intensive care unit. Acta Paediatr . 2016;105(5):e237-e239. doi:10.1111/apa.13369 